\title{
Biological enhancement of graft-tunnel healing in anterior cruciate ligament reconstruction
}

\author{
MARISTELLA F. SACCOMANNO, LUIGI CAPASSO, LUCA FRESTA, GIUSEPPE MILANO \\ Department of Orthopaedics, Catholic University, “A. Gemelli” University Hospital, Rome, Italy
}

\begin{abstract}
The sites where graft healing occurs within the bone tunnel and where the intra-articular ligamentization process takes place are the two most important sites of biological incorporation after anterior cruciate ligament (ACL) reconstruction, since they help to determine the mechanical behavior of the femur-ACL graft-tibia complex. Graft-tunnel healing is a complex process influenced by several factors, such as type of graft, preservation of remnants, bone quality, tunnel length and placement, fixation techniques and mechanical stress. In recent years, numerous experimental and clinical studies have been carried out to evaluate potential strategies designed to enhance and optimize the biological environment of the graft-tunnel interface.

Modulation of inflammation, tissue engineering and gene transfer techniques have been applied in order to obtain a direct-type fibrocartilaginous insertion of the ACL graft, similar to that of native ligament, and to accelerate the healing process of tendon grafts within the bone tunnel. Although animal studies have given encouraging results, clinical studies are lacking and their results do not really support the use of the various strategies in clinical practice. Further investigations are therefore needed to optimize delivery techniques, therapeutic concentrations, maintenance of therapeutic effects over time, and to reduce the risk of undesirable effects in clinical practice.
\end{abstract}

\footnotetext{
Corresponding Author:

Maristella F. Saccomanno, MD

Department of Orthopaedics, Catholic University,

“A. Gemelli” University Hospital

Largo A. Gemelli 8, 00168 Rome, Italy

E-mail: maristellasaccomanno@hotmail.it
}

Keywords: graft-tunnel healing, anterior cruciate ligament reconstruction, biological enhancement, physical stimulation.

\section{Introduction}

In recent years, numerous experimental and clinical studies have been carried out to evaluate the effects of exogenous factors on the healing of a tendon graft within a bone tunnel. These studies focused on two different goals: first, to accelerate tendon-to-bone healing by modulating the inflammatory phase and increasing bone apposition around the tendon graft and new formation of collagen fibers at the graftbone interface (with the dual aim of allowing early postoperative return to normal daily activities as well as sports, and of reducing the risk of graft failure due to pullout from the bone tunnel); and second, to promote differentiation of the newly formed tendonbone junction into a direct-type insertion, as similar as possible to that of normal anterior cruciate ligament (ACL), in order to obtain a more physiological distribution of tensile forces between the intra-articular part of the graft and its insertion on the bone.

\section{Inflammation modulation techniques}

Inflammation is a fundamental phase of the tendonbone healing process, but it can ultimately result in fibrosis rather than healthy regenerated tissue. Hence, the use of biological techniques to moderate inflammation, so as to avoid healing occurring through the formation of fibrosis scar tissue and to facilite osteointegration. Macrophages are among the most important inflammatory cells accumulating around 
the tendon graft from the earliest stage of healing, and they play a critical role in the initiation and regulation of tendon healing (1). Therefore, altering macrophage behavior might result in changes in the tendon-bone healing pattern. Bisphosphonates selectively induce macrophage apoptosis. In a recent study in a rat model, intraperitoneal injections of liposomal clodronate significantly decreased macrophages and transforming growth factor beta (TGF- $\beta$ ) accumulation at the tendon-bone interface, inducing accelerated healing, characterized by enhanced collagen fiber continuity, increased osteoid formation and a greater degree of interface remodeling between tendon and bone at all time points (2). On biomechanical analysis, the experimental group showed significantly greater increases than the control specimens with respect to load to failure $(13.5 \pm 4.2 \mathrm{~N}$ and $9.7 \pm 3.9 \mathrm{~N}$, respectively; $\mathrm{p}<0.05)$ and stiffness $(11.5 \pm 5.0 \mathrm{~N} / \mathrm{mm}$ and $7.5 \pm 3.2 \mathrm{~N} / \mathrm{mm}$, respectively; $\mathrm{p}<0.05$ ) (2). Similarly, Lui et al. (3) showed that continued systemic administration of alendronate reduced peri-tunnel bone resorption and increased mineralized tissue inside the bone tunnel in a rat model. However, a systemic increase in bone mineral density (BMD) was also observed (3). Therefore, the same study group (4) subsequently evaluated the use of a single local administration of low-dose alendronate into the bone tunnel during ACL reconstruction. They observed comparable benefits on the reduction of peri-tunnel bone loss, enhancement of bone tunnel mineralization, tunnel graft integrity, graft osteointegration and mechanical strength of the reconstructed complex at early stage post-reconstruction, but minimal systemic effects.

Moreover, macrophages produce matrix metalloproteinases (MMPs), which play a central role in extracellular matrix remodeling during the healing process. Demirag et al. (5) tested the potential enhancement effect of local injection of $\alpha-2$ macroglobulin on graft-tunnel healing in a rabbit model of ACL reconstruction. The injection of $\alpha-2$ macroglobulin was supposed to block synovial MMP activity. Two and five weeks after the reconstruction, a significantly decreased concentration of type-I collagenase (MMP8) was observed in the synovial fluid when compared with the control group. Histological examination showed that in the treated specimens, compared with the controls, the tendon-bone interface within the tunnel had more areas of denser connective tissue ingrowth. Mechanical testing showed that the mean ultimate failure load of treated specimens was significantly greater than in control specimens at both 2 and 5 weeks.

Furthermore, osteoclast differentiation and activation are provided by the osteoprotegerin (OPG) / receptor activator of nuclear factor-kappa B (RANK) / RANK ligand (RANKL) system (6). Rodeo et al. (7) compared the effect of OPG and RANKL activity on tendon-bone healing in a rabbit model of ACL reconstruction. The Authors showed significantly improved bone formation and stiffness around the tendon graft in the OPG-treated group compared with the RANKL-treated group at 8 weeks after surgery.

Finally, it has recently been reported that statins possess anti-inflammatory effects and can stimulate angiogenesis $(8,9)$. Oka et al. (10) conducted a study in a rabbit model of ACL reconstruction using a hamstring graft implanted with either simvastatin-conjugated gelatin hydrogel or gelatin hydrogel alone in their bone tunnels. They showed that angiogenesis and osteogenesis were greater in the simvastatin-treated group at 2 weeks after surgery. Moreover, smaller tibial bone tunnels were found in the simvastatin treated group on computed tomography (CT) scan at 2 and 4 weeks after surgery. Biomechanical testing at 2 weeks demonstrated a significant increase in the ultimate failure load in the simvastatin-treated group.

\section{Bone proteins and growth factors}

Several studies have shown the effect of polypeptides such as bone morphogenetic proteins (BMPs) and growth factors (GFs) on the activation and regulation of proliferation and differentiation of bone, fibrous and cartilaginous tissues (11-13). On the basis of these experiences, other Authors investigated the role of BMPs and GFs in promoting tendon-to-bone healing through the activation and acceleration of bone ingrowth, collagen fiber synthesis, and fibrocartilaginous differentiation at the tendon-bone graft interface, the aim being to obtain early formation of tendonbone insertion, similar to that of normal ACL.

Recombinant human bone morphogenetic proteins (rhBMPs) are physiological agents responsible for the 
inherent potential of bone to regenerate. They promote the differentiation of early-stage mesenchymal stem cells (MSCs) into chondrogenic and osteogenic lineages that support new bone formation. RhBMP-2 delivery systems, based on collagen gels and sponges, and designed to allow the prolonged and local release of rhBMP-2, have been studied, and found to significantly enhance new bone formation $(14,15)$. Rodeo et al. (16) reported the effect of locally applied rhBMP-2 on the tendon-bone interface in a canine model. They showed that animals treated with rhBMP-2 showed radiographic evidence of more extensive formation and closer apposition of new bone around the tendon graft in comparison with controls. Biomechanical analysis showed higher pullout strength in BMP-treated specimens. Anderson et al. (17) reported an experimental study on ACL reconstruction with an autologous semitendinosus (ST) tendon graft in a rabbit model, using a collagen sponge, wrapped around the portion of the graft inside the bone tunnel, as the carrier vehicle to release a bone-derived extract containing several BMPs (BMP-2, BMP-3, BMP-4, BMP-5, BMP-6 and BMP-7) and GFs with known osteoinductive activity, such as transforming growth factor (TGF) and fibroblast growth factor. Although this use of a mixture of bonederived proteins made it impossible to discriminate the effects of each one on tendon-bone healing, the Authors observed that animals treated with bonederived proteins showed, on histological examination, more consistent and dense interface tissue and closer apposition of new bone to the graft, with the occasional formation of a fibrocartilaginous interface, when compared with control specimens at 2, 4 and 8 weeks after surgery. Biomechanical analysis demonstrated a significant increase in the ultimate tensile strength of the treated grafts (from $47 \%$ more than controls at 2 weeks to $80 \%$ more than controls at 8 weeks). Mihelic et al. (18) reported a study on ACL reconstruction using an autologous peroneus tertius tendon graft in a sheep model. They applied a carrier sponge with rhBMP-7 to the tendon graft -bone interface and observed more extensive bone formation and remodeling around the graft, as well as greater tensile strength in the experimental group than in control knees, at 3 and 6 weeks after surgery. Recent studies have also evaluated the use of injectable calcium phosphate cement (CPC) as a carrier of BMPs. Ma et al. (14) reported that animals treat- ed with injectable CPC-rhBMP-2 showed superior tendon-to-bone healing and a significant increase in the width of new bone formation at the tendon-bone interface. However, the slow degradation rate of injectable $\mathrm{CPC}$ could block the ingrowth of new bone and might delay the osteointegration process (19). Therefore, recombined bone xenograft (RBX), a new type of biosynthetic material which can be directly incorporated into injectable CPC, has been suggested to be more effective in augmenting bone ingrowth in animal models (20). RBX has already been used as a BMP carrier, showing accelerated tendon-bone healing (21).

Moreover, Weimin et al. (22) showed that RBX further accelerated tendon-to-bone healing and provided higher maximum loads to failure when compared with injectable CPC alone after ACL reconstruction in a rabbit model.

With regard to GFs, Yamazaki et al. (23) showed, in a dog model, that TGF- $\beta 1$, mixed with fibrin sealant and applied in the graft-bone gap of the tibial tunnel, enhanced the formation of collagen resembling Sharpey's fibers and provided higher load to failure and stiffness of the graft-tibia complex compared with control knees at 3 weeks after surgery. Sasaki et al. (24) applied granulocyte colony-stimulating factor (G-CSF) around the flexor digitorum superficialis tendon graft used for an extra-articular model of ACL reconstruction in dogs. G-CSF is a member of the cytokine family of GFs, known to stimulate the development of progenitor cells to neutrophils and also to modulate their actions. The Authors showed enhanced angiogenesis and evidence of Sharpey-like fibers at 2 weeks after surgery and increased osteogenesis and biomechanical properties at 4 weeks. Recently, Qin et al. (25) performed a similar study in a rabbit model. TGF- $\beta 1$ was injected directly into the bone tunnel. Histology revealed increased numbers of fibroblasts and collagen fibers at 1,3 and 6 months after surgery compared with control knees. Mechanical properties (maximum force and elastic modulus) differed significantly from those of the control group only at 3 and at 6 months. Instead, Nakase et al. (26) evaluated the effect of hepatocyte growth factor (HGF) on tendon-bone healing in a rabbit model. It is already known that administration of HGF or expression of the HGF gene promotes tissue regeneration or improves pathology in various disease models in different tissues (27). The Authors demon- 
strated that local administration of recombinant HGF into the bone tunnel also accelerated the tendon-bone healing process, both histologically and mechanically. In particular, HGF-treated tendons showed lamellar bone and Sharpey-like fibers as early as 4 weeks after treatment, while a direct-type insertion, characterized by a continuous four-layer structure of bone, calcified cartilage, fibrocartilage and tendon, was regenerated at 12 weeks (26).

Finally, over the past decade, platelet-rich plasma (PRP) has been extensively used in many fields; PRP allows the release of numerous GFs and proteins to the surrounding tissue, thereby accelerating tissue repair after surgery (28). A recent study (29) explored the tendon-bone healing effects of a PRP plus a deproteinized bone compound in a rabbit model of ACL reconstruction. At 4, 8 and 12 weeks after the surgery, the bone density of the tendon-bone junction was higher in the experimental group than in the control knees. Similarly, on biomechanical testing, the maximum tensile load of the experimental group was higher than that of the control group at 4 and 8 weeks.

\section{Clinical studies}

Several studies have investigated the potential beneficial effects, on tendon-bone healing, of PRP administration. Three systematic reviews have recently been published on this topic (30-32). Data pooling was impossible for several reasons: different ACL reconstruction techniques were performed in each trial, different PRP preparations were used, and PRP was administered in different ways (intra-articular injection, combined with scaffolds, around or into the graft, into one or both bone tunnels). However, it appeared that the addition of PRP did not affect bone tunnel healing; furthermore, no differences, versus controls, were found in tendon-bone integration, or in the prevention of bone tunnel enlargement. Only two studies $(33,34)$ showed reduction of edema and increased vascularity at 1 month after surgery.

\section{Cell therapy}

Cell therapy is based mainly on the use of stem cells. Some Authors have investigated the effects of MSCs on the quality and rate of tendon graft osteointegration
(35-39). MSCs are pluripotent cells that can be obtained from various tissues and, under appropriate conditions, can differentiate into tissues of various lineages. Three main sources of MSCs have been investigated with regard to their capacity to enhance tendon-bone healing after ACL reconstruction: bone marrow, tendon, and umbilical cord. MSCs are usually isolated and cultured in vitro, included in a fibrin gel as carrier, and applied along the tendon graft-bone interface. Several studies have investigated the potentiality of bone marrow stem cells in rabbit models using either soft tissue autograft or allograft material (35-37). They showed that, at 8 weeks after surgery, a mature zone of fibrocartilage blending from bone to the allograft, strongly resembling a normal ACL insertion, can be found. Moreover, higher load to failure rates, compared with the control group, were found after injections of bone marrow stem cells into the bone tunnel. Lui et al. (38), in a rat model of ACL reconstruction, investigated the effects of the addition of rat patellar tendon-derived stem cells. The stem cells were sutured to the flexor digitorum longus tendon graft and routed through the bone tunnels. CT scan showed higher tunnel BMD (42.3\% increase, $\mathrm{p}=.047)$ and bone volume/total volume $(\mathrm{BV} / \mathrm{TV})(625 \%$ increase, $\mathrm{p}=.009)$ at the metaphyseal region of the tibial tunnel at week 2 and at the femoral tunnel at week 6 (BMD: 30.8\% increase, $\mathrm{p}=.014$; BV $/$ TV: $100 \%$ increase, $\mathrm{p}=.014)$ compared with the control group. Biomechanical properties were also greater in the experimental group at 2 and 6 weeks after surgery. Jang et al. (39) evaluated the efficacy of transplantation of non-autologous human umbilical cord blood-derived MSCs into the bone tunnel in a rabbit model. No evidence of immune rejections was detected. At 12 weeks, micro-CT showed significantly smaller tibial and femoral bone tunnel enlargement in the treated group; histology showed a smooth transition through fibrocartilage from tendon to bone, resembling the direct insertion of the normal ACL.

\section{Clinical studies}

To the best of our knowledge, only one study (40) has investigated the efficacy of non-cultivated bone marrow MSCs in tendon-bone healing after ACL reconstruction. In this recent study, MSCs were harvested from the anterior iliac crest and centrifuged intraoperatively; $3 \mathrm{ml}$ was then obtained and injected into the 
femoral tunnel and the femoral end of the hamstring graft. Magnetic resonance examination, 3 months later, showed no difference in the signal-to-noise ratio of the fibrous interzone between the experimental group and the conventional ACL reconstruction. Histology of the graft biopsies was also performed in 2 patients (one treated with stem cells and the other not). The results were consistent with imaging findings, showing no difference in the amount of collagen, the vascularity and the number of fibroblasts between the groups. Differences in these results compared with previous animal studies could be related to the absence of cell culture, which clearly provided a higher amount of cells per ml.

\section{Gene therapy}

Limits in the intra-articular use of bone proteins and growth factors include their short half-life and removal by synovial fluid, which can affect the maintenance of therapeutic local concentrations. For this reason, some researchers have attempted to develop gene transfer techniques, which involve the injection of transduced cells that express genes allowing the synthesis of the desired proteins (i.e. GFs) at high local concentrations for prolonged periods of time. Martinek et al. (41) reported a study on a genetically engineered ST tendon graft used for ACL reconstruction in rabbits, conducted to evaluate the capacity of BMP-2 gene transfer to improve tendon-bone healing. At 6 weeks, they observed that grafts infected with adenovirus BMP-2 showed broad zones of newly formed transition layer at the graft-bone interface, resembling a direct-type insertion. Moreover, on mechanical testing, the experimental group showed greater ultimate failure load and stiffness than the controls. However, although these results are encouraging, many safety questions need to be answered and regulatory issues addressed before gene transfer can be proposed as a therapeutic method in orthopaedics (42).

\section{Bone substitutes}

Autogenous bone grafts are probably one of the best options for promoting bone healing because they offer the best osteoconductive, osteogenic and osteoinductive properties, without the risk of immunoreactions or disease transmission. A recent study (43) in a rat model confirmed that within 4 weeks of surgery, bone graft cells migrate to the tendon graft and differentiate into cells involved in collagen production and macrophages, contributing to the early stage of remodeling of the tendon graft. However, disadvantages of autologous bone grafts include potential morbidity at the donor site, the risk of wound infection, increased blood loss, and longer surgical times (44). For these reasons, biosynthetic bone substitutes, such as demineralized bone matrix (DBM), have been considered. DBM consists of insoluble collagen and natural non-collagenous proteins, which are prepared by acid extraction of allograft bone and are thought to promote the early differentiation of MSCs into chondrogenic and osteogenic lineages, leading to new bone formation (45). Lovric et al. (46) evaluated the effects of DBM on tendon-bone healing in a rat model. The Authors showed that DBM increased woven bone formation and enhanced bone remodeling as indicated by histology and micro-CT. Moreover, DBM also appeared to influence biomechanical properties, being associated with a significant increase in peak load to failure of the tendon-bone interface at 4 and 6 weeks postoperatively. Hsu et al. (47) evaluated the effects of DBM in a rabbit model. Immunohistochemical and histological analysis revealed that DBM significantly improved tendon-to-bone integration at 12 weeks, increasing expressions of BMP-2 and vascular endothelial growth factor.

Furthermore, Gulotta et al. (48) evaluated the effect of administration of a magnesium-based bone adhesive into the bone tunnel around the graft in a rabbit model. Application of the bone adhesive resulted in enhanced bone formation and increased load to failure at 6 weeks after surgery.

Nevertheless, several studies have shown that graft healing within bone tunnels can be enhanced by local application of CPC, which is a resorbable and osteoconductive biomaterial (49-55). In rabbit models of ACL reconstruction using CPC-treated autografts, new bone and Sharpey-like fiber formation were observed at the graft-bone interface at 6 and 12 weeks after surgery, respectively $(49,51)$. Recent studies have shown that the osteoconductivity of CPC can be further enhanced by the incorporation of strontium (56, 
57). The Authors showed new bone formation at 3 weeks, indirect healing at 6 weeks, and remodeling into a direct-type insertion at 12 weeks after surgery.

\section{Clinical studies}

Two studies evaluated the efficacy of calcium phosphate and a nanohydroxyapatite-based bone graft substitute, respectively $(58,59)$. Mutsuzaki et al. (58) conducted a prospective randomized clinical trial in 64 patients who underwent a single bundle ACL reconstruction using either a four-strand ST tendon or fourstrand ST and gracilis (GR) tendons with EndoButton femoral fixation and screw washer tibial fixation. The patients were randomized into two groups: one with and the other without CPC around the graft. The CPC-group showed improved anterior knee stability and Lysholm scores at the 2-year follow-up and reduced percentage of bone tunnel enlargement in both tunnels at the 1-year follow-up compared with the conventional reconstruction. Iorio et al. (59) conducted a randomized controlled clinical trial in 40 male patients to evaluate the efficacy of a nanohydroxyapatite bone-based graft in facilitating tendonbone incorporation after ACL reconstruction with hamstrings. Use of the nanohydroxyapatite bone substitute did not provide significant clinical improvements in terms of better knee stability or patient satisfaction. Radiological data showed better results in terms of tendon-bone interface and bone edema remodeling at 1 and 3 months after surgery. At 6 months after surgery the differences were no longer significant.

\section{Biological scaffolds}

In recent years, a number of Authors have focused on the use of periosteal grafts (60-64). Because the periosteum is an osteogenic tissue that modulates bone formation and remodeling at the cortical bone surface, it was hypothesized that it might act as a biological scaffold. Experimental studies on extra-articular models in rabbits showed superior histological and biomechanical features in tendon grafts enveloped in periosteum in the intraosseous part $(60-62,64)$. Moreover, the biological effect of the periosteum on tendon graft bone healing was reported to be more evident when the cambial layer of the scaffold faced the tunnel wall (63), and fresh periosteal graft was reportedly more effective than fresh-frozen graft (60). Subsequently, Chen et al. (65) showed that photoencapsulation of BMP-2 and periosteal progenitor cells improved tendon graft healing at 6 weeks after transplantation of the long digitorum extensor tendon into a bone tunnel in the proximal tibia in a rabbit model. Biomechanical testing revealed higher maximum pullout strength and stiffness at 3 and 6 weeks as compared to controls.

Finally, the use of PRP combined with a canine small intestinal submucosa scaffold has also been tested in a rabbit model (66). The Authors showed a greater cellular response, but lower tensile strength in the PRP group compared with the animals treated with the scaffold only, at 8 weeks after surgery. According to the Authors, the lower biomechanical properties of the PRP group could be due to the intense inflammatory response in the early stages of healing and the persistence of cytokine release (in particular TGF- $\beta 1$ ) at relatively high levels from PRP resulting in extensive lesions due to chondrocyte infiltration of the graft.

\section{Mechanical and physical stimulation}

It has been shown that physical shock wave treatment can induce neovascularization and improve blood supply at the tendon-bone junction $(67,68)$. Wang et al. (69) used a rabbit ACL reconstruction model to show a time-dependent effect of shock waves on the tendon-bone interface. The experimental group was treated with 500 impulses of shock waves at $14 \mathrm{kV}$ for 4 weeks after surgery. Contralateral knees were used as controls. New trabecular bone was seen to be significantly increased around the tendon graft in the treated group at each time interval (from 1 to 24 weeks after treatment). Furthermore, the tensile strength of the tendon-bone interface was significantly higher in the experimental group at 24 weeks.

Another physical modality that has been shown to produce biological effects on bone healing is low intensity pulsed ultrasound (LIPUS). LIPUS was found to stimulate osteoblast proliferation, endochondral ossification, and enhancement of mineralization in vitro (70). Walsh et al. (71) used an intra-articular sheep model of ACL reconstruction to examine the 
effects of LIPUS on tendon-bone healing. LIPUS was applied daily for 20 minutes over the femoral and tibial tunnels for 12 weeks after surgery. Histology showed new bone formation at 3 weeks after surgery, Sharpey like-fibers at 6 weeks, and an accelerated rate of angiogenesis at 3, 6 and 12 weeks after surgery. Stiffness and peak load were greater than in the control group at 26 weeks after surgery.

Yeh et al. tested the efficacy of hyperbaric oxygen (HBO) treatment on tendon-bone healing and its impact on the outcome of ACL reconstruction (72) in a rabbit model. Rabbits were exposed to $100 \%$ oxygen at 2.5 atmospheres of pressure for 2 hours daily, on 5 consecutive days a week, whereas the control group animals were kept in cages exposed to normal air before ACL reconstruction using ST graft. HBO treatment significantly improved neovascularization and progressive interface incorporation of the tendon graft and bone at 12 weeks after surgery. Biomechanical analysis showed that the HBO group achieved higher maximal pullout strength than the control group at 12 and 18 weeks.

\section{Clinical studies}

Wang et al. (73), after showing beneficial effects of extracorporeal shockwave therapy (ESWT) on tendon healing in a rabbit model, recently conducted a randomized clinical study in 53 patients, who underwent a single bundle ACL reconstruction with a hamstring graft. Patients in the experimental group received 1500 impulses of ESWT at $20 \mathrm{kV}$ (equivalent to 0.298 $\mathrm{mJ} / \mathrm{mm}^{2}$ energy flux density) focused on the middle third of the tibial tunnel immediately after surgery. Xray and magnetic resonance evaluation showed a significantly smaller tibial tunnel in the ESWT group at 6 months and 2 years after surgery. However, no differences in BMD were detected between the groups at any time point.

\section{Conclusions}

Considerable efforts have been made in recent years to improve quality and rate of tendon-bone healing. Even though animal studies have given promising results, the limited evidence from human studies does not really show superior results compared with conventional ACL reconstructions.

Further investigations are therefore needed to opti- mize delivery techniques, therapeutic concentrations, and maintenance of therapeutic effects over time, and to reduce the risk of undesirable effects in clinical practice.

\section{References}

1. Kawamura S, Ying L, Kim HJ, et al. Macrophages accumulate in the early phase of tendon-bone healing. J Orthop Res. 2005;23:1425-1432.

2. Hays PL, Kawamura S, Deng XH, et al. The role of macrophages in early healing of a tendon graft in a bone tunnel. J Bone Joint Surg Am. 2008;90:565-579.

3. Lui PP, Lee YW, Mok TY, et al. Alendronate reduced peritunnel bone loss and enhanced tendon graft to bone tunnel healing in anterior cruciate ligament reconstruction. Eur Cell Mater. 2013;25:78-96.

4. Lui PP, Lee YW, Mok TY, et al. Local administration of alendronate reduced peri-tunnel bone loss and promoted graftbone tunnel healing with minimal systemic effect on bone in contralateral knee. J Orthop Res. 2013;31:1897-1906.

5. Demirag B, Sarisozen B, Ozer O, et al. Enhancement of tendon-bone healing of anterior cruciate ligament grafts by blockage of matrix metalloproteinases. J Bone Joint Surg Am. 2005;87:2401-2410.

6. Boyle WJ, Simonet WS, Lacey DL. Osteoclast differentiation and activation. Nature. 2003;423:337-342.

7. Rodeo SA, Kawamura S, Ma CB, et al. The effect of osteoclastic activity on tendon-to-bone healing: an experimental study in rabbits. J Bone Joint Surg Am. 2007;89:2250-2259.

8. Okura H, Asawa K, Kubo T, et al. Impact of statin therapy on systemic inflammation, left ventricular systolic and diastolic function and prognosis in low risk ischemic heart disease patients without history of congestive heart failure. Intern Med. 2007;46:1337-1343.

9. Bitto A, Minutoli L, Altavilla D, et al. Simvastatin enhances VEGF production and ameliorates impaired wound healing in experimental diabetes. Pharmacol Res. 2008;57: 159-169.

10. Oka S, Matsumoto T, Kubo S, et al. Local administration of low-dose simvastatin-conjugated gelatin hydrogel for tendonbone healing in anterior cruciate ligament reconstruction. Tissue Eng Part A. 2013;19:1233-1243.

11. Yasko AW, Lane JM, Fellinger EJ, et al. The healing of segmental bone defects, induced by recombinant human bone morphogenetic protein (rhBMP-2). A radiographic, histological, and biomechanical study in rats. J Bone Joint Surg Am. 1992;74:659-670.

12. DesRosiers EA, Yahia L, Rivard CH. Proliferative and matrix synthesis response of canine anterior cruciate ligament fibroblasts submitted to combined growth factors. J Orthop Res. 1996;14:200-208.

13. Jelic M, Pecina M, Haspl M, et al. Regeneration of articular cartilage chondral defects by osteogenic protein-1 (bone morphogenetic protein-7) in sheep. Growth Factors. 2001;19: 101-113.

14. Ma CB, Kawamura S, Deng XH, et al. Bone morphogenetic proteins-signaling plays a role in tendon-to-bone healing: a study of rhBMP-2 and noggin. Am J Sports Med. 2007;35: 597-604. 
15. Lee KW, Lee JS, Jang JW, et al. Tendon-bone interface healing using an injectable rhBMP-2-containing collagen gel in a rabbit extra-articular bone tunnel model. J Tissue Eng Regen Med. 2015; doi: 10.1002/term.2041.

16. Rodeo SA, Suzuki K, Deng XH, et al. Use of recombinant human bone morphogenetic protein- 2 to enhance tendon healing in a bone tunnel. Am J Sports Med. 1999;27:476-488.

17. Anderson K, Seneviratne AM, Izawa K, et al. Augmentation of tendon healing in an intraarticular bone tunnel with use of a bone growth factor. Am J Sports Med. 2001;29:689-698.

18. Mihelic R, Pecina M, Jelic M, et al. Bone morphogenetic protein-7 (osteogenic protein-1) promotes tendon graft integration in anterior cruciate ligament reconstruction in sheep. Am J Sports Med. 2004;32:1619-1625.

19. Pan W, Wei Y, Zhou L, et al. Comparative in vivo study of injectable biomaterials combined with BMP for enhancing tendon graft osteointegration for anterior cruciate ligament reconstruction. J Orthop Res. 2011;29:1015-1021.

20. Pan W, Hu Y, Wei Y, et al. Recombined bone xenografts enhance tendon graft osteointegration of anterior cruciate ligament reconstruction. Int Orthop. 2009;33:1761-1768.

21. Zhang W, Pan W, Zhang M, et al. In vivo evaluation of two types of bioactive scaffold used for tendon-bone interface healing in the reconstruction of anterior cruciate ligament. Biotechnol Lett. 2011;33:837-844.

22. Weimin P, Dan L, Yiyong W, et al. Tendon-to-bone healing using an injectable calcium phosphate cement combined with bone xenograft/BMP composite. Biomaterials. 2013;34: 9926-9936.

23. Yamazaki S, Yasuda K, Tomita F, et al. The effect of transforming growth factor-beta1 on intraosseous healing of flexor tendon autograft replacement of anterior cruciate ligament in dogs. Arthroscopy. 2005;21:1034-1041.

24. Sasaki K, Kuroda R, Ishida K, et al. Enhancement of tendonbone osteointegration of anterior cruciate ligament graft using granulocyte colony-stimulating factor. Am J Sports Med. 2008;36:1519-1527.

25. Qin J, Hou ZQ, Wang $\mathrm{H}$, et al. Effects of gene-activated matrix on autograft healing of anterior cruciate ligament. Mol Med Rep. 2013;7:679-683.

26. Nakase J, Kitaoka K, Matsumoto K, et al. Facilitated tendonbone healing by local delivery of recombinant hepatocyte growth factor in rabbits. Arthroscopy. 2010;26:84-90.

27. Kitamura K, Iwanami A, Nakamura M, et al. Hepatocyte growth factor promotes endogenous repair and functional recovery after spinal cord injury. J Neurol Res. 2007;85:23322342.

28. Anitua E, Andia I, Ardanza B, et al. Autologous platelets as a source of proteins for healing and tissue regeneration. Thromb Haemost. 2004;91:4-15.

29. Zhai W, Lv C, Zheng Y, et al. Weak link of tendon-bone healing and a control experiment to promote healing. Arch Orthop Trauma Surg. 2013;133:1533-1541.

30. Vavken P, Sadoghi P, Murray MM. The effect of platelet concentrates on graft maturation and graft-bone interface healing in anterior cruciate ligament reconstruction in human patients: a systematic review of controlled trials. Arthroscopy. 2011;27:1573-1583

31. Andriolo L, Di Matteo B, Kon E, et al. PRP Augmentation for ACL reconstruction. Biomed Res Int. 2015;2015:371746.

32. Figueroa D, Figueroa F, Calvo R, et al. Platelet-rich plasma use in anterior cruciate ligament surgery: systematic review of the literature. Arthroscopy. 2015;31:981-988.

33. Vogrin M, Rupreht M, Dinevski D, et al. Effects of a platelet gel on early graft revascularization after anterior cruciate lig- ament reconstruction: a prospective, randomized, doubleblind, clinical trial. Eur Surg Res. 2010;45:77-85.

34. Rupreht M, Vogrin M, Hussein M. MRI evaluation of tibial tunnel wall cortical bone formation after platelet-rich plasma applied during anterior cruciate ligament reconstruction. Radiol Oncol. 2013;47:119-124.

35. Ouyang HW, Goh JC, Lee EH. Use of bone marrow stromal cells for tendon graft-to-bone healing: histological and immunohistochemical studies in a rabbit model. Am J Sports Med. 2004;32:321-327.

36. Lim JK, Hui J, Li L, et al. Enhancement of tendon graft osteointegration using mesenchymal stem cells in a rabbit model of anterior cruciate ligament reconstruction. Arthroscopy. 2004;20:899-910.

37. Soon MY, Hassan A, Hui JH, et al. An analysis of soft tissue allograft anterior cruciate ligament reconstruction in a rabbit model: a short-term study of the use of mesenchymal stem cells to enhance tendon osteointegration. Am J Sports Med. 2007;35:962-971.

38. Lui PP, Wong OT, Lee YW. Application of tendon-derived stem cell sheet for the promotion of graft healing in anterior cruciate ligament reconstruction. Am J Sports Med. 2014;42: 681-689.

39. Jang KM, Lim HC, Jung WY, et al. Efficacy and safety of human umbilical cord blood-derived mesenchymal stem cells in anterior cruciate ligament reconstruction of a rabbit model: new strategy to enhance tendon graft healing. Arthroscopy. 2015;31:1530-1539.

40. Silva A, Sampaio R, Fernandes R, et al. Is there a role for adult non-cultivated bone marrow stem cells in ACL reconstruction? Knee Surg Sports Traumatol Arthrosc. 2014;22:66-71.

41. Martinek V, Latterman C, Usas A, et al. Enhancement of tendon-bone integration of anterior cruciate ligament grafts with bone morphogenetic protein-2 gene transfer: a histological and biomechanical study. J Bone Joint Surg Am. 2002;84-A:11231131.

42. Evans $\mathrm{CH}$, Huard J. Gene therapy approaches to regenerating the musculoskeletal system. Nat Rev Rheumatol. 2015;11:234242.

43. Komiyama H, Arai Y, Kajikawa Y, et al. The fate and role of bone graft-derived cells after autologous tendon and bone transplantation into the bone tunnel. J Orthop Sci. 2013; 18: 994-1004.

44. Dimitriou R, Mataliotakis GI, Angoules AG, et al. Complications following autologous bone graft harvesting from the iliac crest and using the RIA: a systematic review. Injury. 2011;42 Suppl 2:S3-15.

45. Peterson B, Whang PG, Iglesias R, et al. Osteoinductivity of commercially available demineralized bone matrix. Preparations in a spine fusion model. J Bone Joint Surg Am. 2004;86A:2243-2250.

46. Lovric V, Chen D, Yu Y, et al. Effects of demineralized bone matrix on tendon-bone healing in an intra-articular rodent model. Am J Sports Med. 2012;40:2365-2374.

47. Hsu SL, Wang CJ. The use of demineralized bone matrix for anterior cruciate ligament reconstruction: a radiographic, histologic, and immunohistochemical study in rabbits. J Surg Res. 2014;187:219-224

48. Gulotta LV, Kovacevic D, Ying L, et al. Augmentation of tendon-to-bone healing with a magnesium-based bone adhesive. Am J Sports Med. 2008;36:1290-1297.

49. Tien YC, Chih TT, Lin JH, et al. Augmentation of tendonbone healing by the use of calcium-phosphate cement. J Bone Joint Surg Br. 2004;86:1072-1076.

50. Huangfu X, Zhao J. Tendon-bone healing enhancement using 


\section{oints}

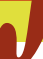

injectable tricalcium phosphate in a dog anterior cruciate ligament reconstruction model. Arthroscopy. 2007;23:455-462.

51. Wen CY, Qin L, Lee KM, et al. The use of brushite calcium phosphate cement for enhancement of bone-tendon integration in an anterior cruciate ligament reconstruction rabbit model. J Biomed Mater Res B Appl Biomater. 2009;89:466474.

52. Shen $\mathrm{H}$, Qiao $\mathrm{G}$, Cao $\mathrm{H}$, et al. An histological study of the influence of osteoinductive calcium phosphate ceramics on tendon healing pattern in a bone tunnel with suspensory fixation. Int Orthop. 2010;34:917-924.

53. Mutsuzaki H, Sakane M. Calcium phosphate-hybridized tendon graft to enhance tendon-bone healing two years after ACL reconstruction in goats. Sports Med Arthrosc Rehabil Ther Technol. 2011;3:31

54. Mutsuzaki H, Sakane M, Fujie H, et al. Effect of calcium phosphate-hybridized tendon graft on biomechanical behavior in anterior cruciate ligament reconstruction in a goat model: novel technique for improving tendon-bone healing. Am J Sports Med. 2011;39:1059-1066.

55. Mutsuzaki H, Sakane M, Nakajima $H$, et al. Calcium phosphate-hybridised tendon graft to reduce bone-tunnel enlargement after ACL reconstruction in goats. Knee. 2012;19:455460.

56. Kuang GM, Yau WP, Lu WW, et al. Local application of strontium in a calcium phosphate cement system accelerates healing of soft tissue tendon grafts in anterior cruciate ligament reconstruction: experiment using a rabbit model. Am J Sports Med. 2014;42:2996-3002.

57. Kuang GM, Yau WP, Lu WW, et al. Use of a strontiumenriched calcium phosphate cement in accelerating the healing of soft-tissue tendon graft within the bone tunnel in a rabbit model of anterior cruciate ligament reconstruction. Bone Joint J. 2013;95-B:923-928.

58. Mutsuzaki H, Kanamori A, Ikeda K, et al. Effect of calcium phosphate-hybridized tendon graft in anterior cruciate ligament reconstruction: a randomized controlled trial. Am J Sports Med. 2012;40:1772-1780.

59. Iorio R, Di Sanzo V, Vadalà A, et al. Nanohydroxyapatitebased bone graft substitute in tunnel enlargement after ACL surgery: RMN study. Clin Ter. 2013;164:e101-106.

60. Ohtera K, Yamada Y, Aoki M, et al. Effects of periosteum wrapped around tendon in a bone tunnel: A biomechanical and histological study in rabbits. Crit Rev Biomed Eng. 2000;28:115-118.
61. Chen $\mathrm{CH}$, Chen WJ, Shih $\mathrm{CH}$, et al. Enveloping the tendon graft with periosteum to enhance tendon-bone healing in a bone tunnel: A biomechanical and histologic study in rabbits. Arthroscopy. 2003;19:290-296.

62. Kyung HS, Kim SY, Oh CW, et al. Tendon-to-bone tunnel healing in a rabbit model: the effect of periosteum augmentation at the tendon-to-bone interface. Knee Surg Sports Traumatol Arthrosc. 2003;11:9-15.

63. Youn I, Jones DG, Andrews PJ, et al. Periosteal augmentation of a tendon graft improves tendon healing in the bone tunnel. Clin Orthop Relat Res. 2004;419:223-231.

64. Chang $\mathrm{CH}$, Chen $\mathrm{CH}$, Liu HW, et al. Bioengineered periosteal progenitor cell sheets to enhance tendon-bone healing in a bone tunnel. Biomed J. 2012;35:473-480.

65. Chen CH, Liu HW, Tsai CL, et al. Photoencapsulation of bone morphogenetic protein-2 and periosteal progenitor cells improve tendon graft healing in a bone tunnel. Am J Sports Med. 2008;36:461-473.

66. Lee AJ, Chung WH, Kim DH, et al. Anterior cruciate ligament reconstruction in a rabbit model using canine small intestinal submucosa and autologous platelet-rich plasma. J Surg Res. 2012;178:206-215.

67. Wang CJ, Huang HY, Pai CH. Shock wave-enhanced neovascularization at the tendon-bone junction: an experiment in dogs. J Foot Ankle Surg. 2002;41:16-22.

68. Wang CJ, Wang FS, Yang KD, et al. Shock wave therapy induces neovascularization at the tendon-bone junction. A study in rabbits. J Orthop Res. 2003;21:984-989.

69. Wang CJ, Wang FS, Yang KD, et al. The effect of shock wave treatment at the tendon-bone interface-an histomorphological and biomechanical study in rabbits. J Orthop Res. 2005; 23:274-280.

70. Ying ZM, Lin T, Yan SG. Low-intensity pulsed ultrasound therapy: a potential strategy to stimulate tendon-bone junction healing. J Zhejiang Univ Sci B. 2012;13:955-963.

71. Walsh WR, Stephens P, Vizesi F, et al. Effects of low-intensity pulsed ultrasound on tendon-bone healing in an intra-articular sheep knee model. Arthroscopy. 2007;23:197-204.

72. Yeh WL, Lin SS, Yuan LJ, et al. Effects of hyperbaric oxygen treatment on tendon graft and tendon-bone integration in bone tunnel: biochemical and histological analysis in rabbits. J Orthop Res. 2007;25:636-645.

73. Wang CJ, Ko JY, Chou WY, et al. Shockwave therapy improves anterior cruciate ligament reconstruction. J Surg Res. 2014;188:110-118. 